\title{
BMJ Global Health Short-term, medium-term and long-term effects of early parenting interventions in low- and middle-income countries: a systematic review
}

To cite: Jeong J, Pitchik HO, Fink G. Short-term, mediumterm and long-term effects of early parenting interventions in low- and middle-income countries: a systematic review. BMJ Global Health 2021;6:e004067. doi:10.1136/ bmjgh-2020-004067

Handling editor Soumyadeep Bhaumik

- Additional material is published online only. To view, please visit the journal online (http://dx.doi.org/10.1136/ bmjgh-2020-004067).

Received 28 September 2020 Revised 21 January 2021 Accepted 10 February 2021

Check for updates

C Author(s) (or their employer(s)) 2021. Re-use permitted under CC BY-NC. No commercial re-use. See rights and permissions. Published by BMJ.

For numbered affiliations see end of article.

Correspondence to

Dr Joshua Jeong;

jjeong@mail.harvard.edu

\section{ABSTRACT}

Introduction Parenting interventions during early childhood are known to improve various child development outcomes immediately following programme implementation. However, less is known about whether these initial benefits are sustained over time.

Methods We conducted a systematic literature review of parenting interventions in low- and middle-income countries (LMICs) that were delivered during the first 3 years of life and had completed a follow-up evaluation of the intervention cohort at least 1 year after the primary postintervention endpoint. We summarized intervention effects over time by child-level and parent-level outcomes as well as by timing of follow-up rounds in the short-term (1-3 years after programme completion), medium-term (4-9 years), and long-term (10+ years). We also conducted exploratory meta-analyses to compare effects on children's cognitive and behavioral development by these subgroups of follow-up rounds.

Results We identified 24 articles reporting on seven randomised controlled trials of parenting interventions delivered during early childhood that had at least one follow-up study in seven LMICs. The majority of follow-up studies were in the short-term. Three trials conducted a medium-term follow-up evaluation, and only two trials conducted a long-term follow-up evaluation. Although trials consistently supported wide-ranging benefits on early child development outcomes immediately after programme completion, results revealed a general fading of effects on children's outcomes over time. Short-term effects were mixed, and medium-term and long-term effects were largely inconclusive. The exploratory meta-analysis on cognitive development found that pooled effects were significant at postintervention and in the short-term (albeit smaller in magnitude), but the effects were not significant in the medium-term and long-term. For behavioural development, the effects were consistently null over time. Conclusions There have been few longer-term follow-up studies of early parenting interventions in LMICs. Greater investments in longitudinal intervention cohorts are needed in order to gain a more comprehensive understanding of the effectiveness of parenting interventions over the life course and to improve the design of future interventions so they can have greater potential for achieving and sustaining programme benefits over time.

\section{Key questions}

What is already known?

- Parenting interventions during early childhood are effective for improving early child development outcomes immediately following programme implementation.

- Although a few individual parenting interventions have demonstrated longer-term benefits on certain child development outcomes, a systematic review of this literature has not previously been conducted.

What are the new findings?

- Our review identified seven randomised controlled trials of parenting interventions that were delivered during early childhood and conducted at least one follow-up of the intervention trial cohort in a lowand middle-income country.

- We found a general fading of intervention impacts on children's development outcomes over time, with mixed results for short-term effects and largely inconclusive results for medium-term and long-term effects.

What do the new findings imply?

- Additional follow-up evaluations are needed to gain a fuller understanding of the short-term, medium-term and long-term effects of early childhood parenting intervention and to inform the design of improved interventions that can maximize and sustain gains in child development outcomes over the life course.

\section{INTRODUCTION}

Globally $43 \%$ of children under 5 years are failing to attain their developmental potential due to poverty, poor health and inadequate stimulation. ${ }^{1}$ The first 3 years of life are a particularly sensitive period of brain and social development, during which parents are the primary providers of care for young children. ${ }^{2}$ Parenting interventions during the earliest years of life are effective for improving a wide range of outcomes. ${ }^{3}$ For example, reviews of common parenting interventions during early childhood—such as psychosocial 
stimulation, ${ }^{45}$ dialogical reading ${ }^{6}$ and attachment interventions ${ }^{7}$ - have consistently revealed positive effects on children's cognitive, language, motor and behavioural development outcomes, as well as parenting knowledge, practices and parent-child interactions immediately after the completion of the intervention. ${ }^{8}$

Parenting interventions during early childhood have received increasing policy attention globally. ${ }^{9}$ Policymakers and researchers have argued that if interventions can positively affect children's cognitive and socioemotional development in the short run during the formative years of early childhood, then such effects may place children on more positive lifelong trajectories to offset the adverse effects of poverty and promote child development over the life course. In addition to the potential for reducing life course inequalities, it has been argued that early life interventions are worth scaling up because they can greatly reduce governmental support needs in the long run. ${ }^{10}$ Yet, experimental evidence to support longerterm effectiveness is in fact scarce.

Much of the limited literature on follow-up effects of early parenting interventions, and early interventions more broadly, comes from high-income countries (HICs). ${ }^{11}$ The majority of these follow-up studies have been small efficacy trials, targeted to vulnerable or at-risk populations (eg, families facing psychosocial risks $^{12}$ or preterm infants ${ }^{13}$ ) and limited to short-term follow-up studies showing some sustained benefits during preschool or middle childhood. Only a few trials have shown persisting long-term benefits on select adolescent or adult development outcomes. ${ }^{14} 15$ The acclaimed adult economic payoffs for investing in early childhood programmes have primarily emerged from two small-scale studies in the United States: the Abecedarian Project ${ }^{16}$ and the Perry Preschool project. ${ }^{17}$ However, as more studies have become available, diminishing intervention impacts have been observed over time, suggesting that general claims of the longer-term benefits of early interventions may be overestimated. ${ }^{18}$

Despite the considerable number of parenting interventions that have been evaluated in low-income and middle-income countries (LMICs), ${ }^{35}$ there have been far fewer follow-up studies in LMICs as compared with HICs. One of the oldest and most prominent examples is the Jamaica Home Visiting programme. In a small efficacy trial, 127 undernourished infants under age 2 years from poor neighbourhoods of Kingston, Jamaica, were randomly assigned to receive weekly home visits from nurses and one of four interventions over a 2-year period: psychosocial stimulation, nutritional supplementation, stimulation and supplementation, or standard healthcare services. ${ }^{19}$ This intervention cohort has been followed to date across childhood, adolescence and early adulthood, with results revealing sustained benefits of the early stimulation intervention on adolescent and early adult outcomes, such as higher educational attainment, reduced depression and higher earnings at the age of 22 years. ${ }^{21}{ }^{21}$ The positive results from this small efficacy study have been widely cited in support of investing and making policy decisions about the potential of scaling up early parenting interventions in LMICs.

Our study aimed to contextualise and synthesise these findings with the emerging body of follow-up studies. We review the literature on parenting interventions delivered during the first 3 years of life in LMICs that also completed at least one subsequent follow-up evaluation. We summarise intervention characteristics, follow-up study designs and intervention effects over time on a broad and inclusive range of child and parent outcomes. Finally, we highlight the implications of our findings with regard to the design, implementation and evaluation of future parenting interventions, and discuss possible strategies for sustaining programme benefits over the life course.

\section{METHODS \\ Search strategy}

A systematic literature review was conducted and reported according to the Preferred Reporting Items for Systematic Reviews and Meta-Analysis guidelines. The methods were prespecified and documented in a protocol (PROSPERO number CRD42020199665). Six electronic bibliographic databases (MEDLINE, Embase, PsycINFO, CINAHL, Web of Science and Global Health Library) were searched for peer-reviewed, published articles from database inception until 18 July 2020 . A string of search terms combined keywords for concepts relating to parenting, early childhood development (ECD), randomised controlled trials (RCTs) and LMICs (online supplemental table S1). A similar search strategy was used for a separate review investigating the immediate effects of parenting interventions on ECD and parenting outcomes. ${ }^{8}$ Reference lists of relevant studies were scanned for any additional studies that may have been missed.

\section{Selection criteria}

Full-text, peer-reviewed articles in English were included if they met the following criteria: (1) parenting interventions that aimed to improve caregiver interactions, behaviours, knowledge, beliefs, attitudes or practices with their children in order to primarily improve ECD; (2) targeted caregivers and young children during the period of early childhood or specifically preconception through the first 3 years of life; (3) interventions evaluated using a randomised controlled study design; (4) had at least one follow-up study that was conducted at least 1 year after the primary postintervention endpoint; and (5) measured a developmental outcome in one of the follow-up studies. Studies were excluded if they met any of the following criteria: (1) represented interventions that did not focus on parenting for ECD; (2) targeted a population of children who were, on average, older than 36 months (eg, preschoolers and school-aged children); or (3) did not conduct a follow-up assessment after the primary postintervention evaluation round. 


\section{Data extraction}

Two reviewers (JJ and research assistant) independently screened the titles and abstracts of each study identified in the systematic search. Full texts of selected studies were reviewed to assess eligibility. Reference lists of included studies and previous reviews were examined to identify any potentially relevant publications not found through the electronic search. Any discrepancy between the reviewers was resolved through discussion and consensus.

Two reviewers (JJ and HOP) independently extracted data from each eligible study using a structured form. The main categories of data that were extracted for each study included characteristics of the sample, intervention details, timeline of follow-up, outcome measures used in follow-up studies and findings over time. Follow-up studies and results were organised according to the original trial. Any discrepancies were resolved through discussion and consensus.

\section{Outcomes}

The primary outcomes focused on measures of child development, which included children's cognitive, language, motor, executive functioning, and socioemotional and behavioural outcomes. Secondary outcomes included any other child-level outcomes over the life course, including education, physical and mental health, and economic productivity. We also considered parentlevel outcomes, such as parenting behaviours or parental depressive symptoms. We aimed to be as inclusive as possible in our review of secondary outcomes.

\section{Risk of bias assessment}

Two reviewers (IJ and HP) independently assessed risk of bias in included studies using the Cochrane Collaboration Risk of Bias Tool. Categorical ratings of high, low or unclear were assigned with regard to random sequence generation, blinding of participants and personnel, incomplete outcome data and selective reporting in each study. Any discrepancies were resolved through discussion and consensus.

\section{Data synthesis}

The main results for each trial were summarised in a table that described the original intervention, the number of follow-up studies, the relevant outcome measures assessed and the intervention effects for each outcome. The outcomes and specific measures used across all studies were summarised. Intervention effects were narratively synthesised by type of outcomes (ie, child-level or parentlevel outcomes) and timing of follow-up (ie, short-term (1-3 years), medium-term (4-9 years) or long-term (10+ years)). In multiarm trials, we focused on the main effect of the parenting intervention. For example, with studies that used $2 \times 2$ factorial cluster RCT design, we compared the two arms that received the parenting intervention to the other two that did not receive the parenting intervention. Due to the varied nature of timing of follow-up studies and outcomes assessed, we primarily conducted a descriptive synthesis of results.
We conducted exploratory meta-analyses on any child development outcome that was repeatedly measured by at least two trials across two out of the three follow-up time frames. As applicable, we calculated effect sizes or the standardised mean differences (SMDs) in the outcome between the parenting intervention and control arms divided by the pooled SD. We reported the effect sizes for each study across follow-ups. Using a stratified random effects meta-analysis model, we explored subgroup differences in the pooled effect sizes by timing of follow-up (ie, postintervention, short-, medium- and long-term). We descriptively compared pooled effects over time by magnitude of estimates rather than statistical testing between subgroups, given the limited number of studies. Figures illustrating the effect sizes across studies and follow-up time points were created in R. Meta-analyses and forest plots were conducted in Stata V.16.

\section{Patient and public involvement}

Patients or the public were not involved in the design and conduct of this research.

\section{RESULTS}

\section{Search result details}

The structured search identified 6620 unique records, and we found an additional 15 relevant articles through scanning references and one article that was published after the search was conducted and identified based on authors' personal knowledge (online supplemental figure S1). Ultimately, a total of 24 articles met the inclusion criteria. These articles corresponded to seven unique intervention cohorts.

\section{Intervention and implementation characteristics}

Table 1 presents details of the intervention content, setting, duration, intensity and original study designs of the seven RCTs included in the review. All seven interventions targeted mothers and incorporated components of psychosocial stimulation to enhance engagement in play and early learning activities or responsive caregiving to improve ECD outcomes. Three of the trials also provided nutritional support (supplementation and/or infant feeding education ${ }^{19}{ }^{22-24}$; one was embedded into an existing cash transfer programme ${ }^{22}$; and one was delivered as part of routine child health visits. ${ }^{25}$ Collectively, the interventions were conducted in seven countries: Jamaica, Uganda, Colombia, South Africa, Pakistan and a multisite study in the Caribbean (Jamaica, Antigua and St. Lucia). Publication dates of original trial results ranged from 1991 to 2017. Enrolled sample sizes of the trials ranged from $127^{19}$ to $1411 .^{24}$ Three of the seven RCTs used a $2 \times 2$ factorial design with stimulation, nutrition and combined intervention arms in addition to a control arm (with one study including a non-stunted population control group), ${ }^{1922} 24$ whereas the remaining four trials tested an intervention against a control group (with one study including an additional normal birthweight control group). The original interventions 


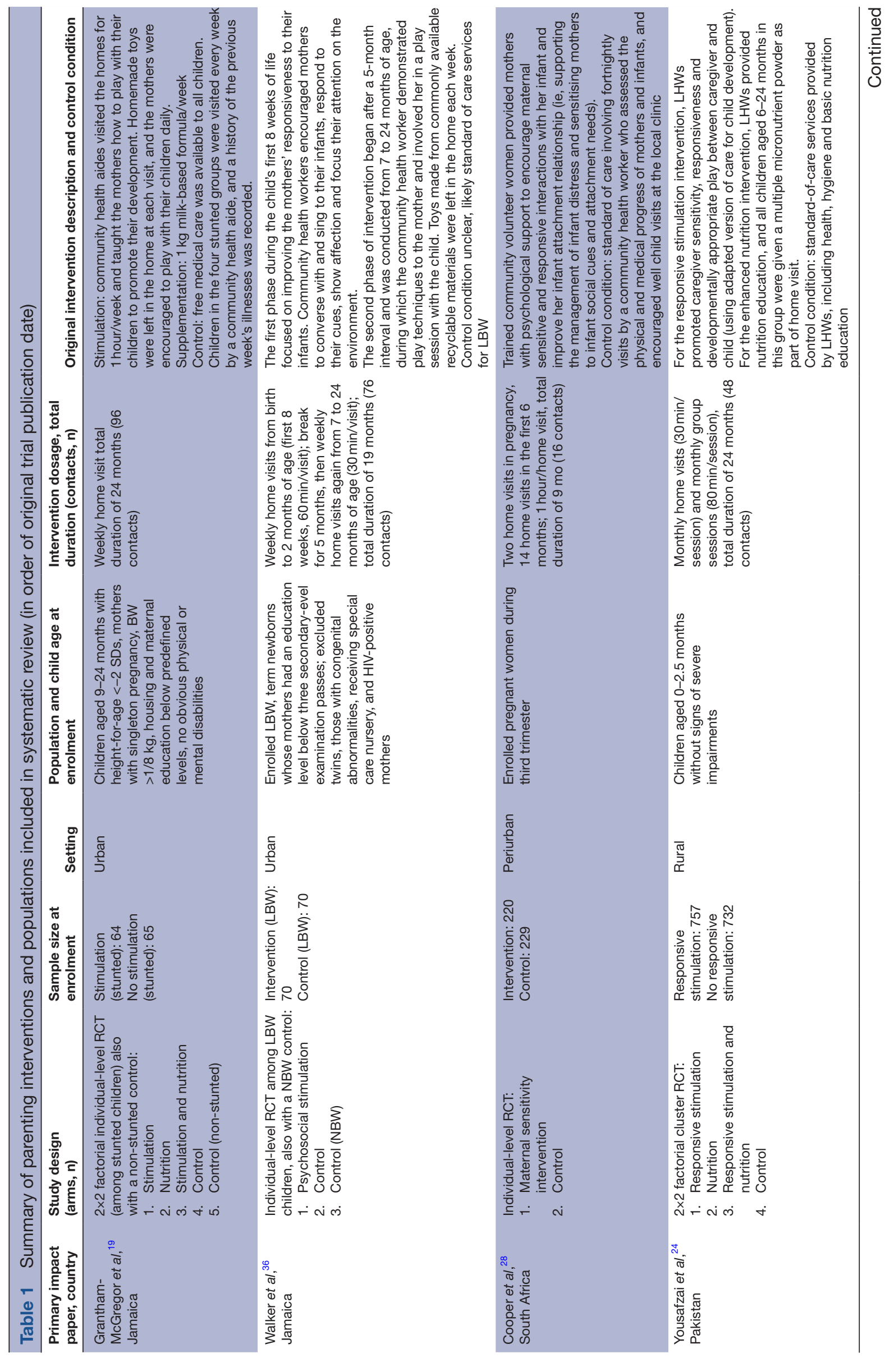




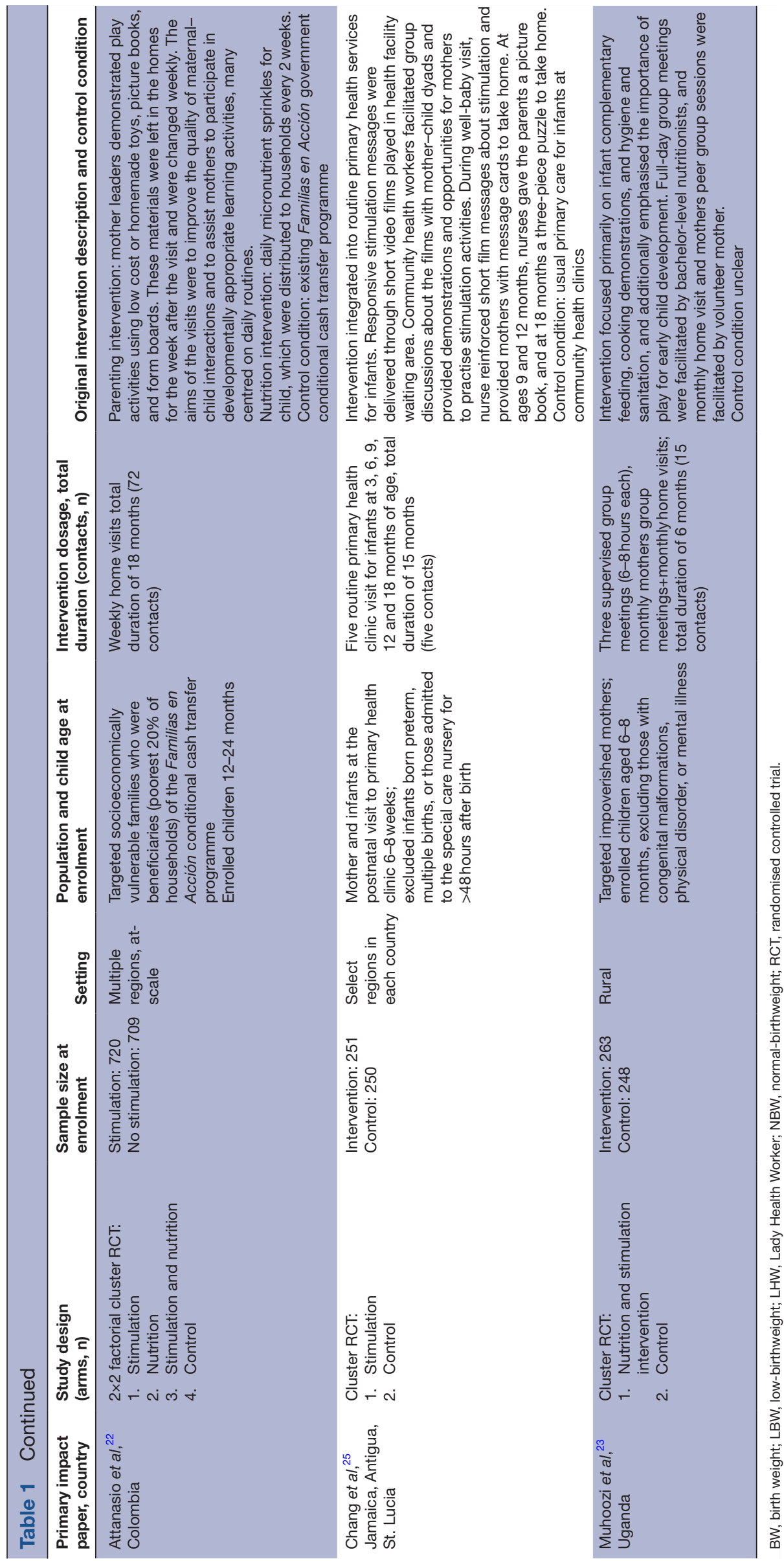



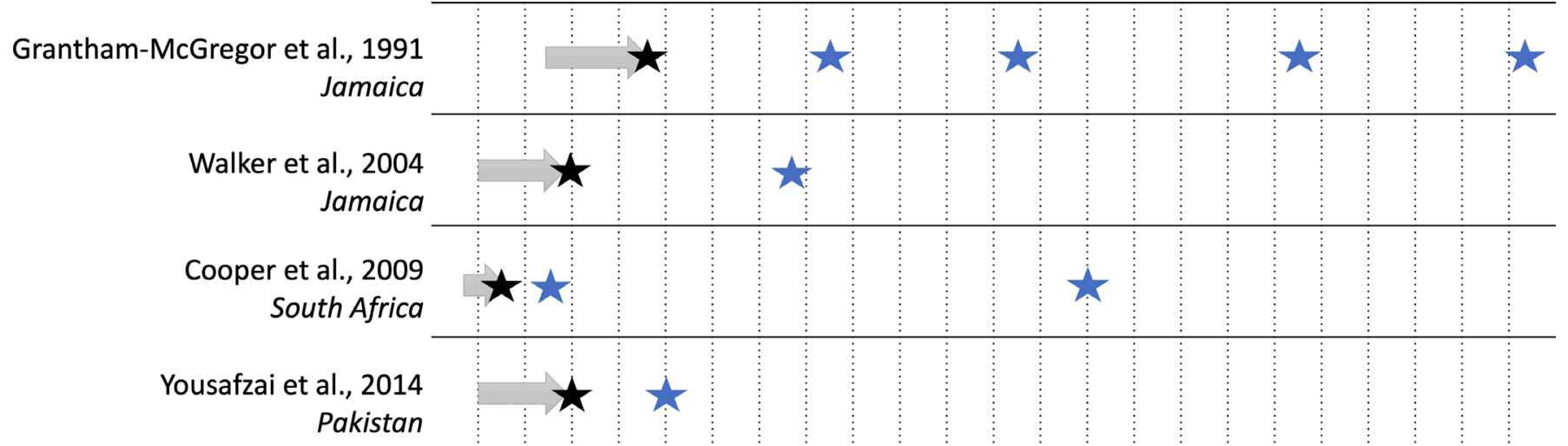

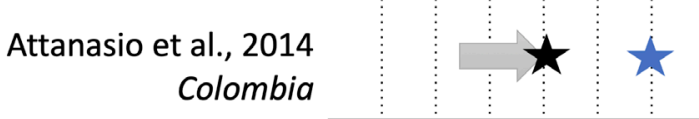

Chang et al., 2015

Jamaica, Antigua, St. Lucia

\author{
Muhoozi et al., 2017 \\ Uganda
}

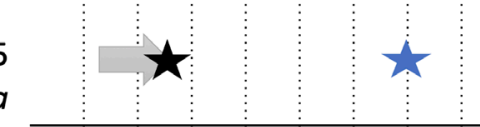

Average child age (years)

Figure 1 Evaluation rounds of included parenting interventions. Note: Arrows represent intervention timing and duration. Black stars represent postintervention evaluation. Blue stars represent follow-up evaluations.

began between pregnancy and 18 months of age and lasted between 6 and 24 months (figure 1). Intervention delivery occurred through weekly contacts in two trials, approximately biweekly contacts in three trials, in two stages of weekly home visits after a 5-month break in one trial, and during five routine health visits over 15 months in one trial. The total programme contacts ranged from $5^{25}$ to 96 contacts. ${ }^{19}$

Risk of bias across the trials was generally low for blinding of outcome assessors and selective reporting across studies (online supplemental table S2). Risk of bias for allocation concealment and random sequence generation for the original trials was mostly unclear. Incomplete outcome data particularly for the follow-up studies were high in half of the trials. Given the nature of psychoeducational and behavioural parenting interventions that involve parents' active participation, blinding of participants was not possible.

\section{Follow-up study details}

The seven trials included in this review correspond to a total of 11 follow-up evaluations that were conducted one or more years after an immediate postintervention evaluation. Figure 1 presents the number and timing of follow-up evaluations for each of the included trials. The numbers of follow-up rounds per trial were one (five trials), two (one trial) and four (one trial). Four trials conducted short-term follow-ups with assessments 1-3 years after intervention completion; three had medium-term follow-ups 4-9 years after intervention completion; and two covered long-term follow-ups $10+$ years after intervention completion. The oldest mean age at follow-up was 22.6 years. The proportion of the original sample revisited during the follow-ups ranged from $29 \%$ (a random subset by design) ${ }^{26}$ to $98 \%^{27}$ (table 2).

\section{Measurement}

Developmental outcomes and assessment tools varied substantially across studies and depending on child age (table 2). Cognitive development or IQ was the most commonly assessed outcome (assessed at 10 follow-ups), followed by behavioural or socioemotional (assessed at 9 follow-ups). The next most common assessments were language (six follow-ups), school readiness, achievement or academic outcomes (five follow-ups) and motor development (three follow-ups). For the long-term follow-ups of the original trial by Grantham-McGregor et $a l^{19}$ (at ages 18 and 22 years), a range of other outcomes were assessed in addition to youth developmental skills, such as mental health symptoms, other behaviours (eg, parenthood and substance use) and earnings.

Fewer parent-level outcomes were assessed in follow-up studies. Eight of 11 follow-ups assessed at least one parent-level outcome. Parenting practices was evaluated in five follow-ups, as was parental depressive symptoms (although one study did not report results in text). Other parent-level outcomes were assessed once (eg, motherchild interactions and self-efficacy) (table 2). 


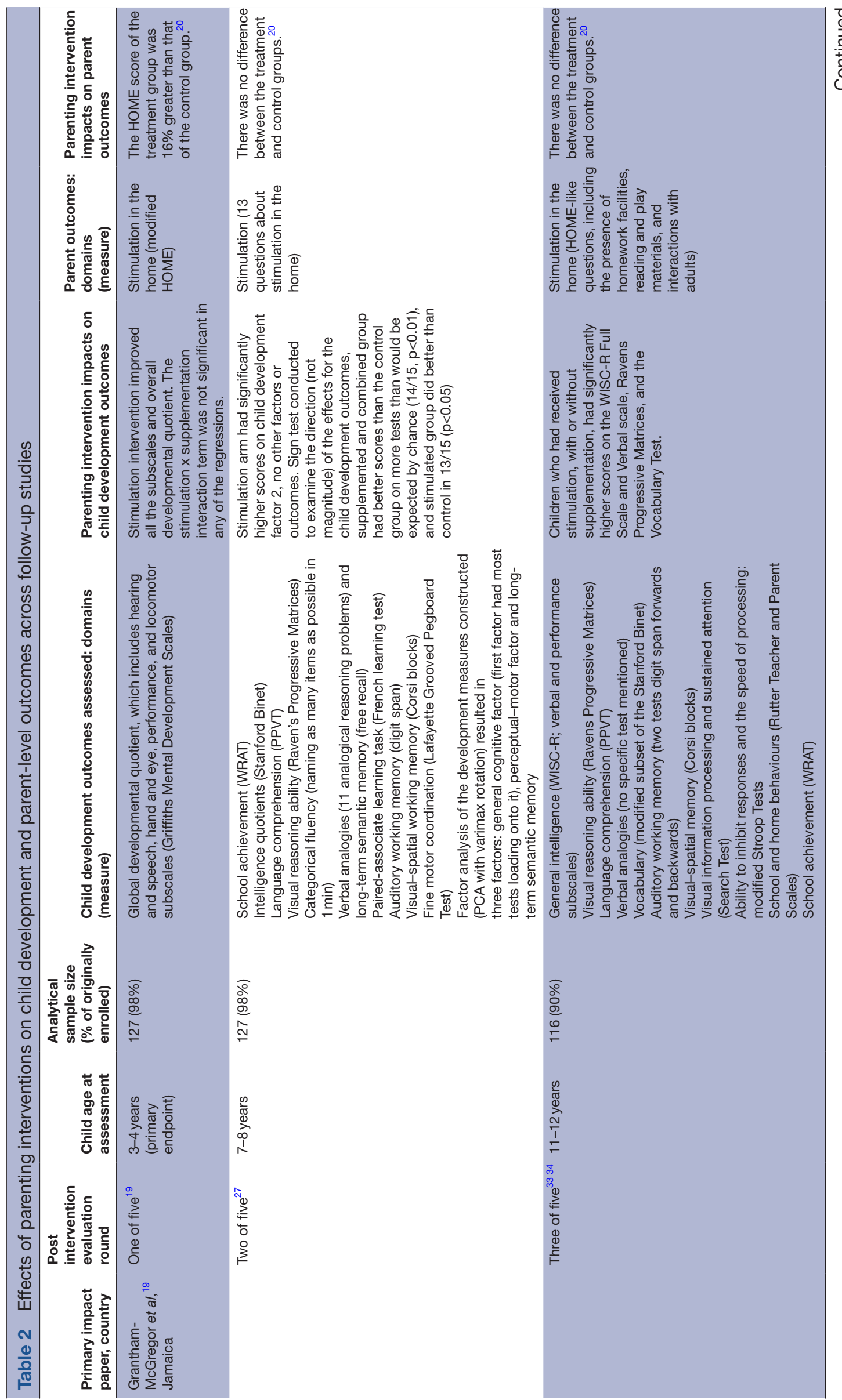




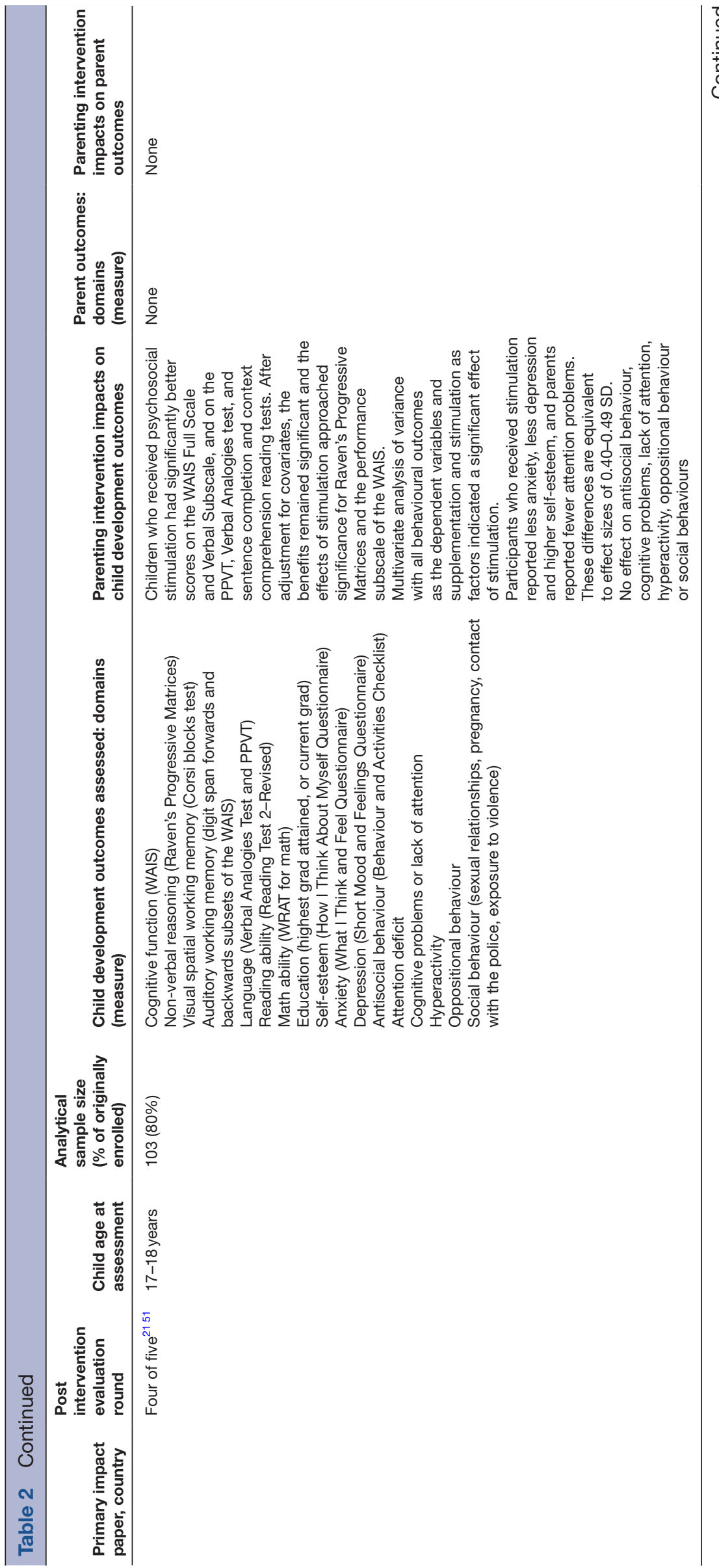




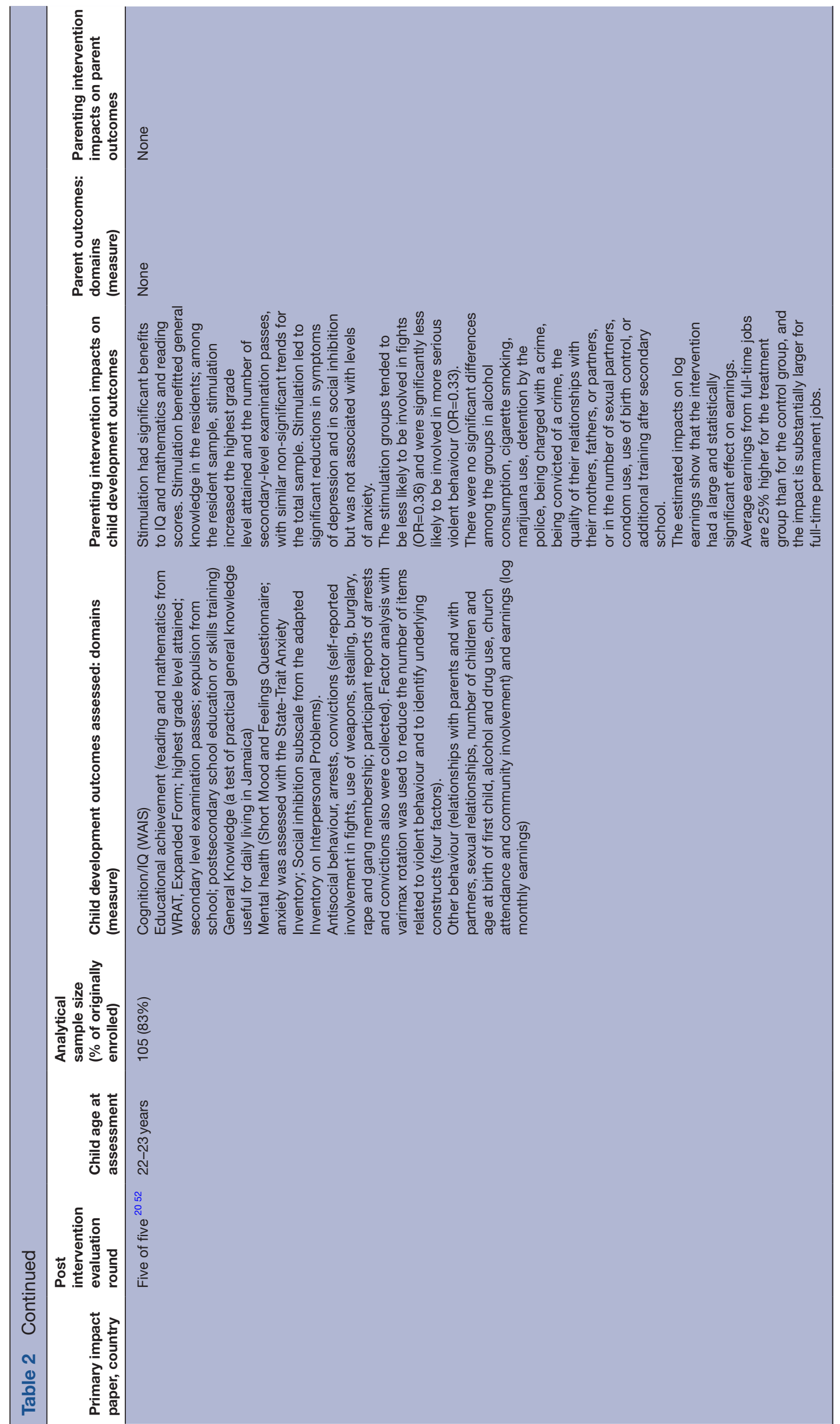




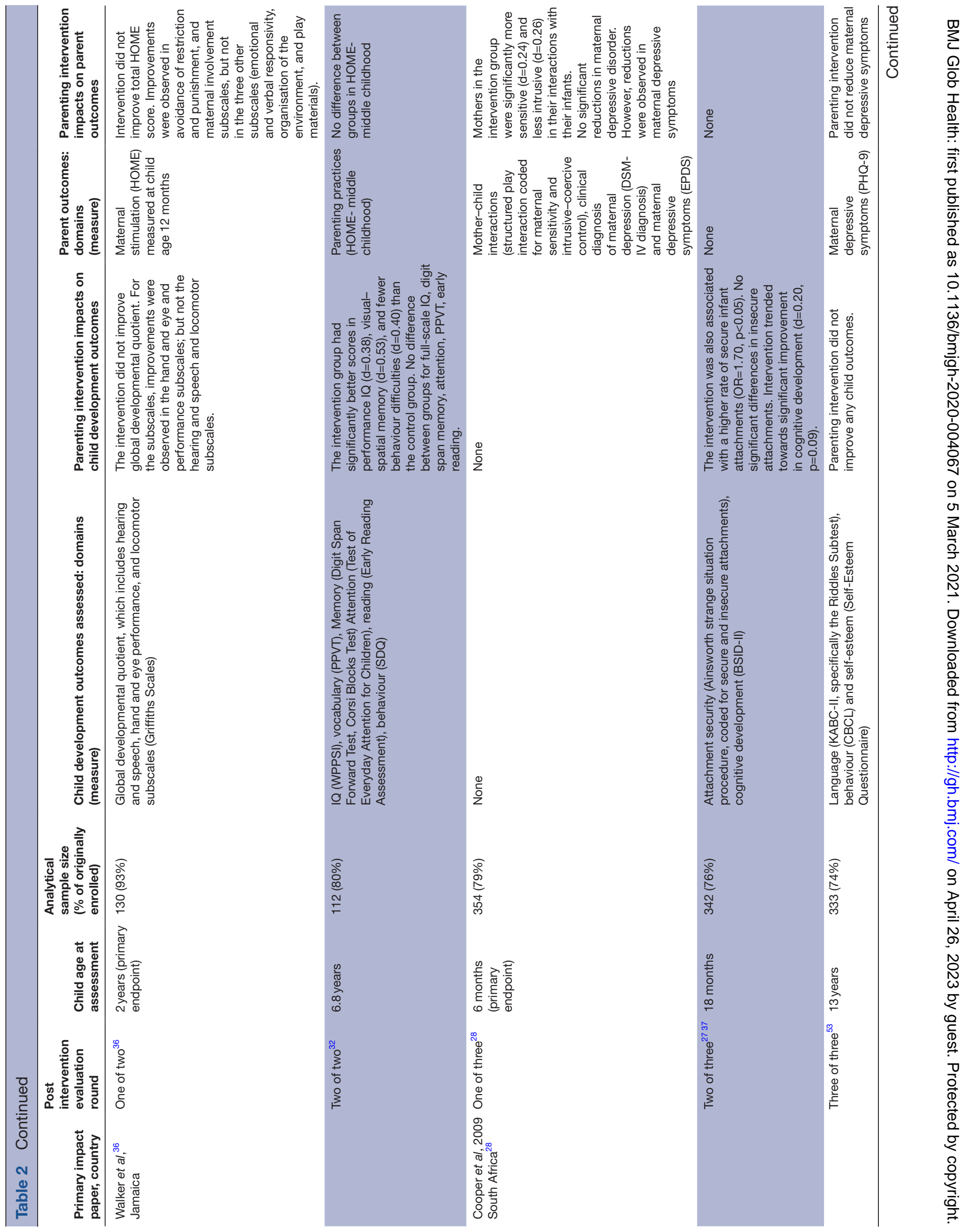




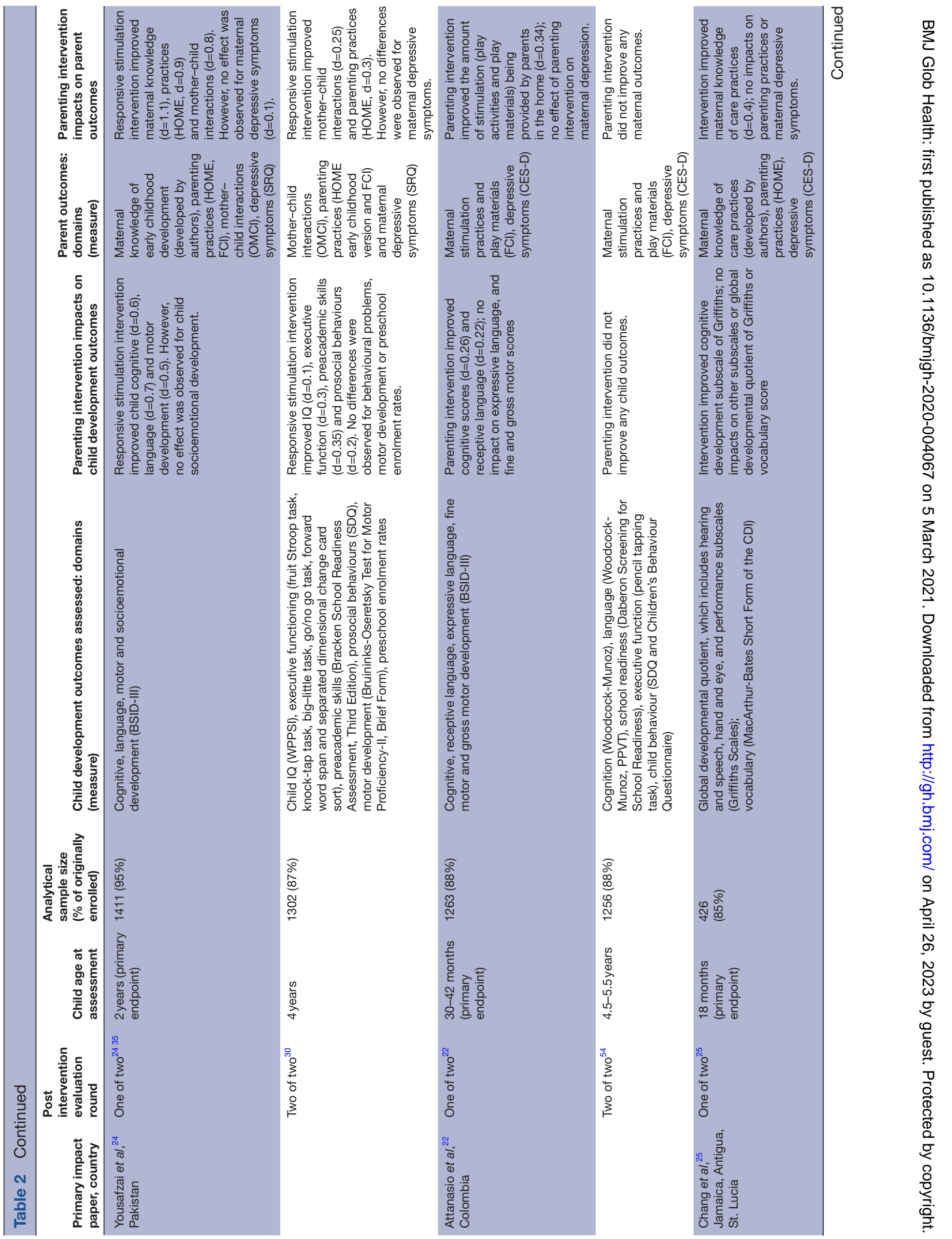




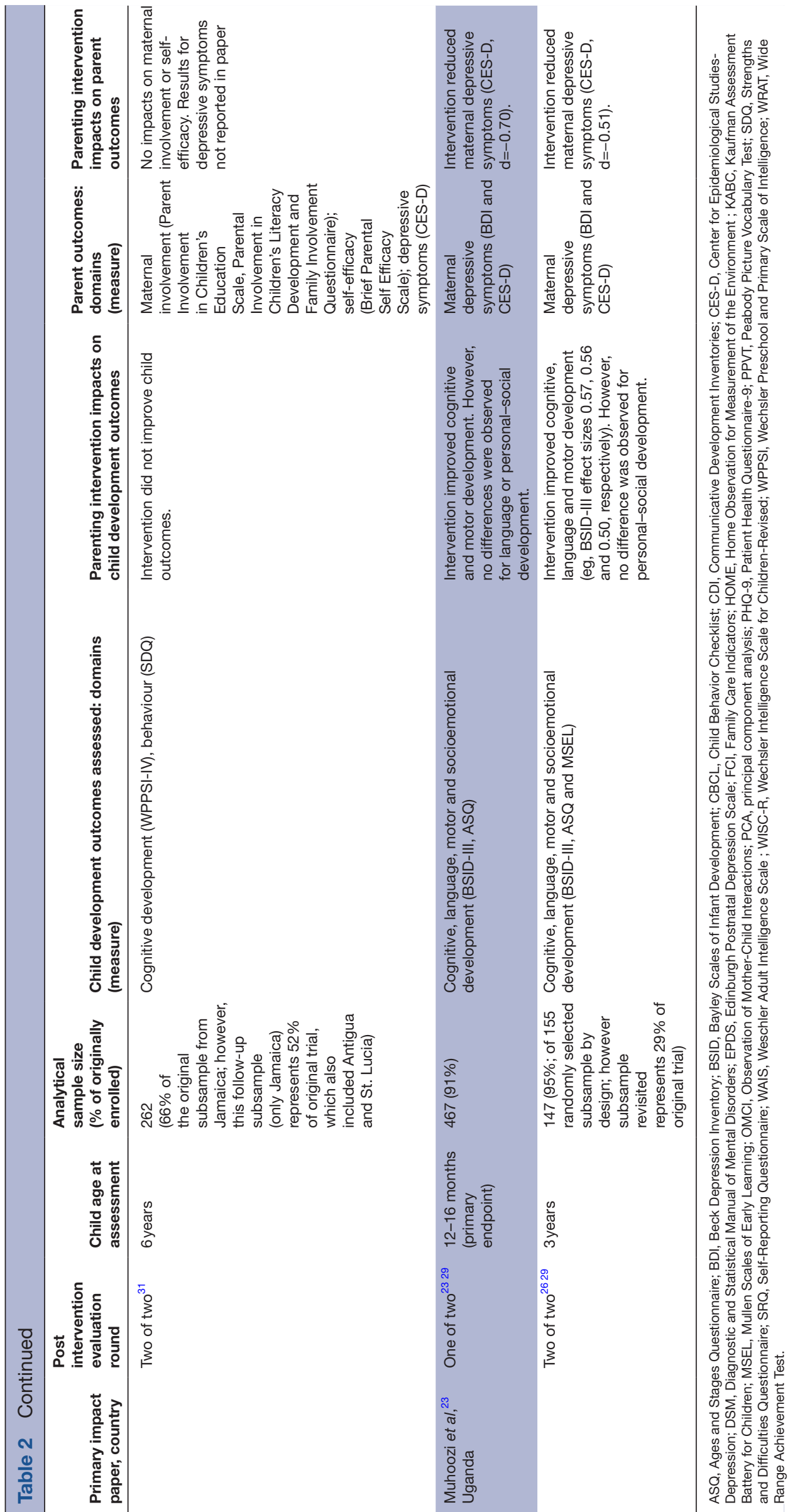




\section{Intervention results over time}

Intervention results are presented here by the timing of evaluation rounds: postintervention and short-term, medium-term and long-term effects. For postintervention results, six of the seven studies assessed immediate impacts on child development, and all found intervention improvements in at least one ECD outcome. Postintervention impacts ranged from relatively small impacts on only certain ECD outcomes in some studies to medium-to-large impacts on all ECD outcomes assessed. For example, Attanasio $e t a l^{22}$ found small improvements in only cognition and receptive language but no significant impacts on expressive language, and fine and gross motor scores. On the other hand, Grantham-McGregor et al found large impacts on the global score, as well as all subscales of the Griffiths Mental Development Scales. The intervention by Cooper $e t a l^{28}$ did not evaluate an ECD outcome at endline but observed improvements in mother-infant interactions.

Four interventions conducted a short-term follow-up in the first 1-3 years following intervention endline. Of these, three found sustained intervention improvements on at least one ECD outcome. One year after intervention endline (at 18 months of age), Cooper et al found infants in the intervention arm had a higher odds of secure attachment, though no significant differences were found for cognitive development. In Uganda, Atakunda et $a l^{2629}$ reported that the intervention improved cognitive, language and motor development, but not socioemotional development, at 2 years following intervention endline (age 3 years), and reduced maternal depressive symptoms. In Pakistan, 2 years postintervention endline (age 4 years), Yousafzai $e t a l^{30}$ found that children in the responsive stimulation group had sustained higher IQ, executive function, preacademic skills and prosocial behaviours, but no differences in behaviours problems, motor development and preschool enrolment rates, compared with those who did not receive responsive stimulation. Mother-child interactions and parenting practices were also sustained in the responsive stimulation group, but no differences were observed in maternal depressive symptoms. In contrast, the follow-up to the trial by Attanasio et al did not find any sustained effects on ECD (ie, cognitive, language, school readiness, executive function and child behaviour) or maternal outcomes (ie, maternal stimulation and depressive symptoms) at 2 years postintervention (age 5 years).

Three interventions conducted a medium-term follow-up evaluation between 4 and 9 years after intervention completion. In the 4.5-year follow-up to the study by Chang et $a l^{25}$ (age 6 years), Smith $e t a l^{31}$ found no effects on the two measured child outcomes (cognitive abilities or socioemotional difficulties) or the two measured parent outcomes (parent involvement and parental selfefficacy). In their 5 year follow-up (age 6.8 years), Walker et $a l^{32}$ found sustained intervention benefits for a few select outcomes: higher scores on child performance IQ and visual spatial memory subscales, and significant reductions in behavioural difficulties. However, there were no treatment differences for the remaining majority of outcomes: full-scale IQ digit span memory, attention, PPVT or early reading. The original trial by GranthamMcGregor et al had two medium-term follow-up studies 4 and 8 years after intervention completion. In the 4-year follow-up (7-8 years), Grantham-McGregor $e t a l^{27}$ found no differences in any child outcome measures when comparing those that were randomised to the stimulation arm compared with the control (10+ outcome measures). After combining all ECD outcomes through a data-driven factor analysis, they found impacts on one of three factors (ie, perceptual-motor factor score). However, in the 8-year follow-up (11-12 years), Walker et $a l^{33}$ found sustained intervention improvements in 4 of the 12 child cognitive outcomes, and Chang et a ${ }^{34}$ did not find improvements in behavior or school achievement. Neither intervention found any medium-term sustained improvements in maternal stimulation in the home.

Two interventions conducted a long-term follow-up 10 or more years after the end of the intervention. Children from the intervention in South Africa by Cooper et alwere followed up 12.5 years after the end of the intervention (age 13 years). Adolescents' language, behaviour and self-esteem outcomes were assessed, as well as maternal depressive symptoms, but there were no intervention differences in any of these outcomes. Children from the intervention in Jamaica by Grantham-McGregor et al were reassessed 14 and 18 years following the primary endpoint. At the 14-year follow-up (age 17-18 years), adolescents randomised to the stimulation intervention had sustained gains in cognitive and language development, academic skills, as well as less anxiety, fewer depressive symptoms and higher self-esteem. No differences were observed for several other outcomes (eg, social, antisocial and hyperactivity behaviours). At the 18-year follow-up (age 22-23 years), persisting intervention benefits were observed in youth IQ and log monthly earnings, and less depression, violent behaviours and involvement in fights. No differences were seen for various other health behaviour (eg, smoking, alcohol and contraceptive use), education and crime outcomes. Parental outcomes were not assessed at either of the follow-ups at 14 or 18 years.

\section{Illustrative examples and exploratory meta-analyses for impacts on cognitive and behavioural development over time}

Cognitive development was the most frequently measured outcome across trials and follow-up rounds. An illustrative comparison of the follow-up effects on cognitive development is presented in figure 2. Eleven follow-up studies across all seven trials evaluated cognitive development outcomes. Two of the four short-term follow-ups demonstrated sustained benefits on cognitive development, whereas the other two studies did not find any significant short-term benefits. Of the four medium-term follow-ups, Grantham-McGregor et alfound that the initial intervention effect on cognitive development faded out after 4 years but resulted in a significant difference after 


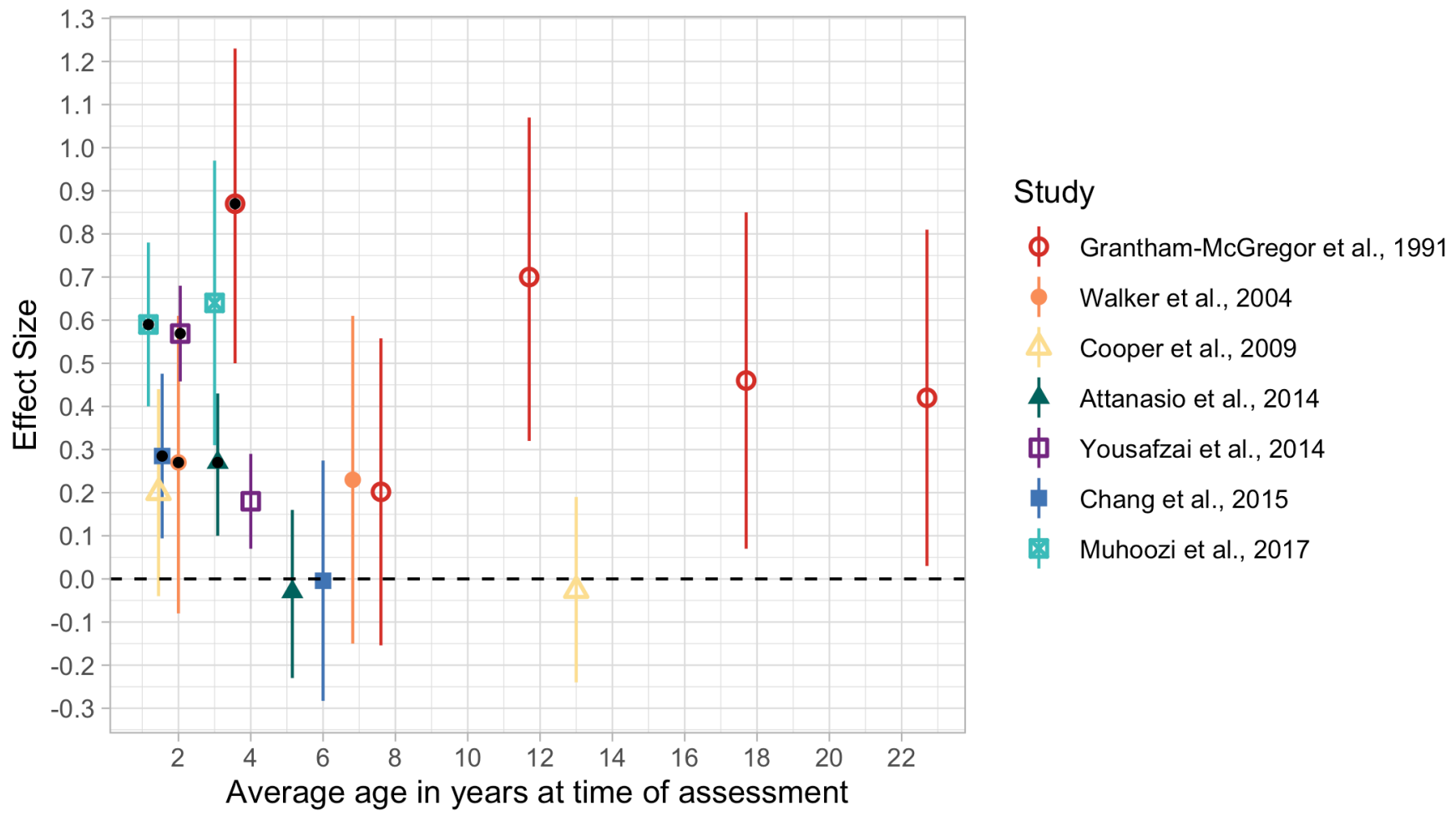

Figure 2 Parenting intervention effects on cognitive development outcomes for each trial across follow-up studies. Note: markers with black dots represent immediate postintervention trial results. For Cooper et al, ${ }^{28}$ there was no postintervention assessment of cognitive development.

8 years. In the other two follow-ups in Jamaica by Walker et $a l$ and Smith et al, there were no benefits observed for either trial after 5 years. Finally, with regard to long-term follow-up results, the trial by Grantham-McGregor et al found sustained improvements on cognitive development after 14 and 18 years, whereas Cooper et al found null effects again after 12.5 years. The second most commonly evaluated outcome across trials and follow-ups was behavioural development in eight studies across all seven trials. With the exception of significant improvements after 5 years in the trial by Walker et al, there were no differences observed in behavioural development in any of the individual follow-up studies (online supplemental figure S2).

Exploratory meta-analyses-stratified by follow-up period (ie, postintervention, short-term, medium-term and long-term effects) - are presented for cognitive and behavioural development outcomes in figures 3 and 4, respectively. See online supplemental table S3 for specific outcome measures used from each study. Results indicated a robust positive postintervention effect for cognitive development ( $\mathrm{SMD}=0.46$ ) that generally faded out over time, with the magnitude of the pooled effect reduced by $41 \%$ to $52 \%$ of the postintervention pooled effect size (figure 3 ). Although there was a small pooled effect on cognitive development in the short-term ( $\mathrm{SMD}=0.21)$, the pooled effects were not significant in medium-term or long-term. For child behaviour, results revealed no detectable pooled effects postintervention or in the short-term, medium-term, or long-term. Although the magnitude of the pooled effects appears to increase for medium-term and long-term results, the individual trials that measured child behaviour in longer-term follow-ups did not also measure postintervention effects on behaviour, which precludes assessment of the magnitude of fade-out effects for this outcome over time.

\section{DISCUSSION}

Our systematic review identified seven RCTs of parenting interventions that conducted a follow-up evaluation of the original trial cohort. Follow-ups were mostly shortterm, within 1-3 years after programme completion; only two trials had long-term follow-ups $(10+$ years $)$ that tracked cohorts from early childhood into adolescence or young adulthood.

Although there were consistent intervention benefits on multiple ECD and parent-level outcomes immediately after programme completion, follow-up results revealed a general fading of effects over time across all trials. The sustainability of intervention effects over time appeared to be associated with the magnitude of immediate postintervention effects on ECD outcomes. For example, with cognitive development, immediate impacts ranged from small effect sizes in four of the studies $(\mathrm{SMD}=0.2-0.3)$ to medium-to-large effect sizes for the remaining three studies $(\mathrm{SMD}=0.5-0.9)$. The three trials with larger immediate postintervention impacts showed significant sustained benefits in the short-term, ${ }^{19} 2324$ whereas the other trials with small postintervention impacts did not show sustained benefits at any subsequent follow-up 


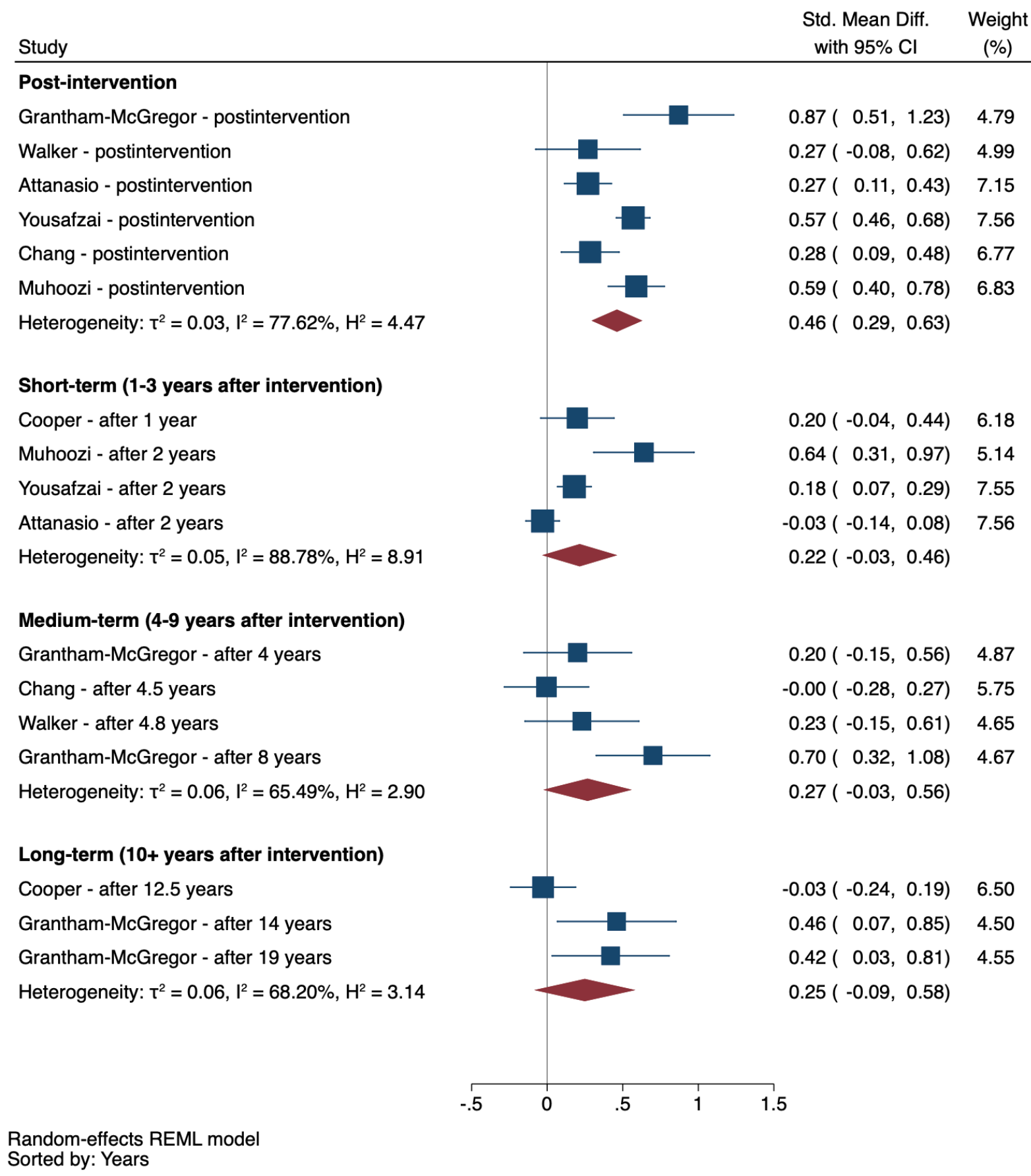

Figure 3 Short-term, medium-term and long-term pooled effects of parenting interventions on cognitive development outcomes. Note: REML, random-effects meta-analysis.

evaluation for any outcomes. Our results suggest there may be a threshold of immediate gains requiredperhaps to the magnitude of at least moderate-sized postintervention effects $(\mathrm{SMD}>0.5)$-in order to activate the potential for longer-term sustained benefits on ECD. Additional follow-up studies with larger samples are needed to confirm these trends, especially considering the wide CIs associated with most estimates.

The two interventions that achieved medium-to-large immediate gains in caregiving and parent-level outcomes were those that similarly had larger postintervention effects on ECD and subsequently sustained short-term benefits on ECD. More specifically, Yousafzai $e t a l^{55}$ found large initial effects on maternal knowledge of ECD, stimulation and mother-child interactions, and sustained benefits on ECD and parent outcomes in the short-term. The trial by Muhoozi et al found medium-sized initial reductions in maternal depressive symptoms and sustained reductions in depression and improvements in ECD outcomes in the short-term. ${ }^{29}$ On the other hand, Attanasio et $a l,{ }^{22}$ Cooper $e t a l,{ }^{28}$ Chang et $a l^{25}$ and Walker $e t a l^{36}$ found small, if any, postintervention effects on maternal 


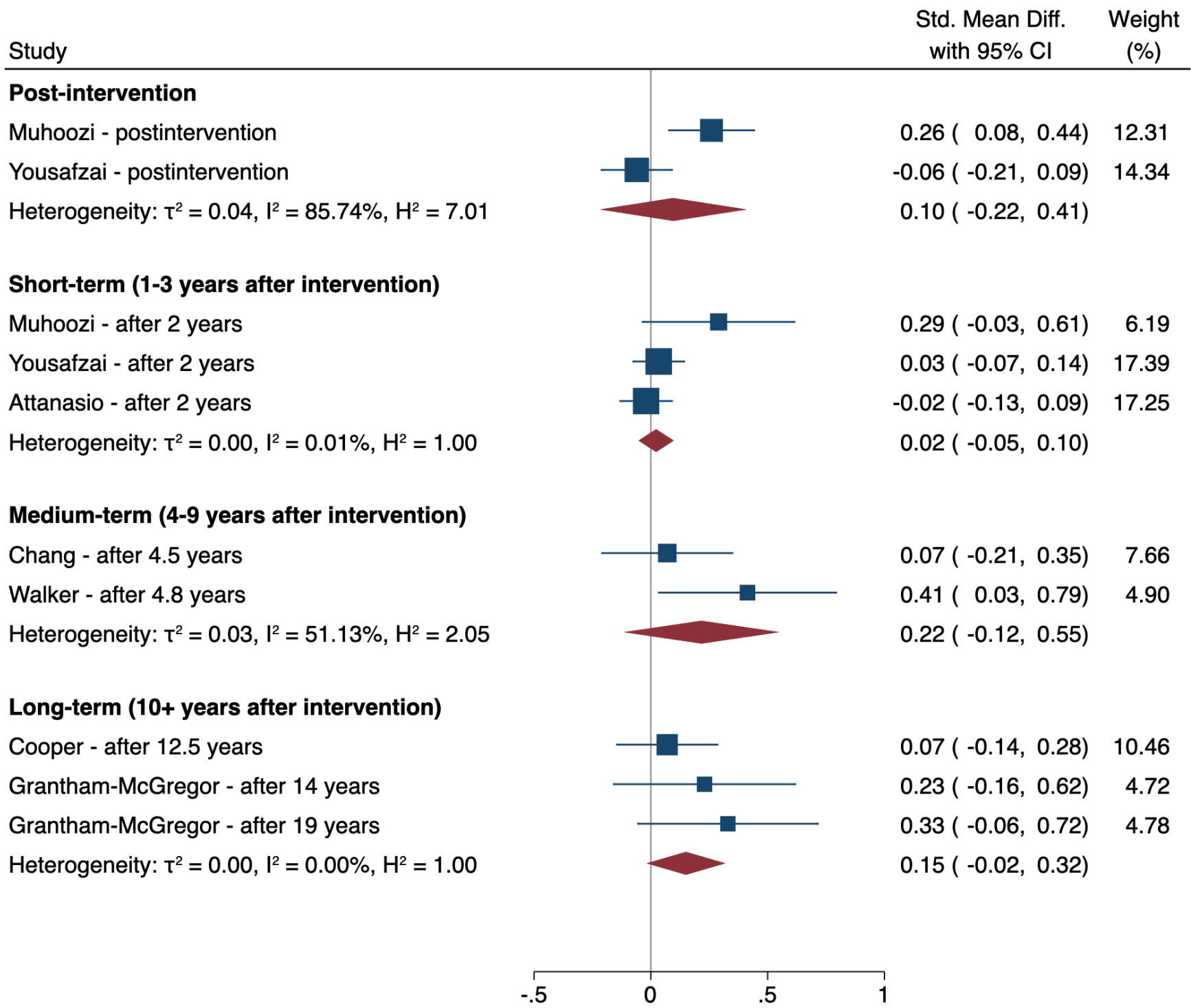

Random-effects REML model Sorted by: Years

Figure 4 Short-term, medium-term and long-term pooled effects of parenting interventions on behavioural outcomes. REML, random-effects meta-analysis.

outcomes and no follow-up effects on any maternal or ECD outcomes over time. Given that improvements in parenting are generally the primary pathway through which these interventions improve child outcomes, ${ }^{4}$ if parenting behaviours are not meaningfully improved postintervention, then fadeout effects on ECD outcomes are even more likely. Our results highlight the importance of targeting and sufficiently improving parental behaviours and well-being in order to sustain longerterm programme impacts on ECD outcomes beyond the completion of parenting interventions.

While we identified a potential trend between initial impacts on ECD and parenting outcomes and sustainability of intervention effects of time, there are also a number of other factors that may explain the heterogeneity in follow-up results. First, intervention theories of change and target populations varied across trials. For example, half of the programmes enrolled birth cohorts and included components to enhance maternal sensitivity and responsiveness beginning during the postnatal period, ${ }^{24} 2836$ compared with other interventions that focused primarily on increasing cognitive stimulation, distributed play materials to the households every week as part of the programme, and more directly engaged a broader and older age range of children between 9 and 24 months at enrolment. ${ }^{19} 22$ Variations in programme components and theories of change may reasonably explain why certain interventions did not improve particular ECD outcomes (eg, no impact of postnatal maternal sensitivity intervention on later child cognitive development outcomes ${ }^{37}$ ) and the null overall effects observed for behavioural development, which may require alternative 
interventions that have a stronger focus on social learning theory. ${ }^{38}$ The majority of interventions concluded prior to child age 2 years, with the exception of Attanasio et $a l^{22}$ that supported children up until age 3 years, and Grantham-McGregor $e t a l^{19}$ that also engaged some children older than 3 years of age, depending on their initial age at enrolment. The transition to preschool is a critical developmental period, during which continued support for parents and children may confer additional advantages that may produce sustained effects on later outcomes. $^{39}$

Second, intervention implementation characteristics also varied substantially in terms of dosage, duration, delivery agents and scale. For example, the original Jamaica Home Visiting programme was the most intensive and involved weekly 1-hour home visits for 24 months, delivered by community health aides, among a small sample in a relatively contained geographical area in the capital city, ${ }^{19}$ compared with a programme in Colombia that was much larger, integrated at scale into the existing conditional cash transfer programme and delivered by volunteer mothers through weekly home visits for 18 months. ${ }^{22}$ It has been suggested that more frequent and longer programme durations are associated with greater immediate postintervention effects of early childhood interventions. ${ }^{40}$ It is likely that sufficient programme exposure, as well as quality implementation, is even more crucial in order to produce longer-term enduring effects. In spite of these trends, it is worth noting that the intervention in Uganda, which had the shortest duration of 6 months, found sustained improvements in ECD outcomes and reductions in maternal depressive symptoms after a 2-year follow-up. ${ }^{26}{ }^{29}$ These unique findings may be explained by the fact that this was primarily a research study (ie, outside of existing community service delivery platform) and used bachelor-level session facilitators that were likely substantially better trained and more skilled than lay community members used in other trials.

Third, characteristics of the study population and context varied widely. For example, the trials in Jamaica targeted stunted and low-birthweight children, and the trials in Colombia and Uganda targeted poor households. Prior studies have suggested that disadvantaged children may be more likely to benefit from early interventions. ${ }^{41}$ Others have suggested that interventions for disadvantaged children may increase likelihood of observing programme effects considering their additional vulnerabilities and already likely delayed developmental trajectories in the absence of any early intervention. ${ }^{42}$ At the same time, broader population-level socioeconomic deprivations can also undermine the sustainability of programme gains. For example, weak community health services, food insecurity or the lack of access to preprimary school education in low-income contexts can compromise the environments needed to subsequently sustain gains in children's developmental skills. ${ }^{43}$

Taken together, our results highlight several gaps and considerations for future research. First, the majority of trials were relatively small efficacy studies, greatly limiting the ability to detect smaller effects in longer-term follow-ups. Moreover, many outcomes assessed in the follow-up rounds were not theoretically justified, and few parent-level outcomes were measured in the follow-up studies. Yet, behavioural changes in caregiving knowledge, skills and practices with their young child are a key theoretical pathway of parenting interventions. ${ }^{27}$ Our results emphasise the need for developing and applying theories of change to investigations of follow-up effects, which can inform decisions about which outcomes to assess and ensure hypothesised mechanisms are adequately captured.

Few trials have conducted post hoc analyses of potential mediators underlying intervention follow-up effects. Of notable exception, the trial in Pakistan found that sustained improvements in maternal scaffolding skills explained benefits of the intervention on children's intelligence and executive functioning, ${ }^{44}$ and sustained improvements in maternal and paternal stimulations explained sustained intervention benefits to children's cognitive and socioemotional development outcomes. ${ }^{45}$ Improved measurement of parenting outcomes across follow-ups and longitudinal mediation analyses are needed to understand common mechanisms that drive sustained treatment gains and identify processes that can be harnessed in future parenting interventions to increase the potential for longer-term impacts.

Although the current evidence for intervention effects on child or parent outcomes is limited in the short-term and even moreso inconclusive in the longer term, it is worth mentioning two additionally plausible interpretations of the present findings. Prior studies have suggested 'sleeper' effects with regard to potential long-term effects of parenting interventions. ${ }^{18}{ }^{46}$ Sleeper effects refer to a phenomenon whereby an intervention produces no immediate postintervention effect (or a small effect) that is latent in the short-term, requires time to fully materialise and then gradually appears at a later follow-up. ${ }^{1847}$ In addition, there may be potential effects that are not being captured using the current measures or for outcomes that were not assessed. Both of these possibilities support continued rounds of follow-up studies in order to explore whether sleeper or unmeasured effects might be a possible explanation for mixed short-term and seemingly null medium-term impacts. The trajectory of follow-up results from the GranthamMcGregor et al study indicated a large immediate postintervention impact, a null medium-term impact, but then a rebounding and sustained positive long-term effect. Based on these results, it appears possible that treatment impacts may fluctuate in the short-term to medium-term. Therefore, multiple waves of follows-ups are needed in order to determine longer-term patterns and potential trajectories of treatment effects.

There are several limitations of our review that are worth highlighting. First, longitudinal trials are often subject to loss to follow-up. The prevalence of loss to 
follow-up among the sample revisited by design ranged from $2 \%$ to $34 \%$. Although some studies stated no observed differences between those who were reassessed and those who were lost to follow-up, others did not specify and therefore results may be subject to bias. Second, as already mentioned, most included trials were relatively small efficacy studies that did not present power calculations to determine whether the sample size was sufficient to detect follow-up treatment effects, which complicates interpretation of null results. Third, many studies did not report quantitative values for each stated outcome or provide details regarding measurement adaptation, reliability and validity. Fourth, quantitative data synthesis for effects over time on cognitive and behavioural development were exploratory in nature. Given the few trials represented and the heterogeneity in interventions, outcome measures and timing, pooled estimates should be interpreted with caution. Finally, our study only included published articles, which introduces the potential for possibly overestimating long-term effects, considering how initial null or weak findings are less likely to conduct follow-up evaluation and be disseminated by authors (ie, publication bias).

\section{CONCLUSIONS}

The findings from our systematic review reveal a dearth of follow-up evaluations of parenting interventions in LMICs. Although parenting interventions have shown robust, wide-ranging immediate postintervention benefits on ECD and parenting outcomes, our review suggests that there is currently limited evidence of sustained short-term impacts and inconclusive evidence regarding medium-term or long-term effects based on only two small efficacy trials. Additional follow-up evaluations are needed to provide a fuller picture of the potential medium-term and long-term intervention effects. In conclusion, parenting interventions during early childhood should not be seen as a 'silver bullet', especially in the contexts of poverty and other psychosocial stressors. Future parenting intervention should consider other types of multicomponent interventions, such as fatherinclusive parenting programmes ${ }^{49}$ or parental mental health promoting interventions, ${ }^{50}$ which may have more transformative benefits to the family environment, and potentially in turn sustain programme benefits for child and parent outcomes over time. Ultimately, accessible and high-quality services for children, parents and families and continued support through complementary interventions are critical for ultimately improving population health and development across the life course.

\footnotetext{
Author affiliations

${ }^{1}$ Department of Global Health and Population, Harvard University T.H. Chan School of Public Health, Boston, Massachusetts, USA

${ }^{2}$ Division of Epidemiology, School of Public Health, University of California, Berkeley, Berkeley, California, USA

${ }^{3}$ Department of Epidemiology and Public Health, Swiss Tropical and Public Health Institute, Basel, Basel-Stadt, Switzerland
}

${ }^{4}$ University of Basel, Basel, Switzerland

Twitter Joshua Jeong @joshuadjeong

Contributors JJ conceived the study, designed the protocol, conducted the database search, reviewed studies for eligibility, conducted the analyses and drafted the manuscript. JJ and HOP extracted the data and created the visualisations. JJ, HOP and GF contributed to the interpretation of data and critical revision of the manuscript and approved the final draft.

Funding The authors have not declared a specific grant for this research from any funding agency in the public, commercial or not-for-profit sectors.

Competing interests None declared.

Patient consent for publication Not required.

Provenance and peer review Not commissioned; externally peer reviewed.

Data availability statement All data relevant to the study are included in the article or uploaded as supplementary information.

Supplemental material This content has been supplied by the author(s). It has not been vetted by BMJ Publishing Group Limited (BMJ) and may not have been peer-reviewed. Any opinions or recommendations discussed are solely those of the author(s) and are not endorsed by BMJ. BMJ disclaims all liability and responsibility arising from any reliance placed on the content. Where the content includes any translated material, BMJ does not warrant the accuracy and reliability of the translations (including but not limited to local regulations, clinical guidelines, terminology, drug names and drug dosages), and is not responsible for any error and/or omissions arising from translation and adaptation or otherwise.

Open access This is an open access article distributed in accordance with the Creative Commons Attribution Non Commercial (CC BY-NC 4.0) license, which permits others to distribute, remix, adapt, build upon this work non-commercially, and license their derivative works on different terms, provided the original work is properly cited, appropriate credit is given, any changes made indicated, and the use is non-commercial. See: http://creativecommons.org/licenses/by-nc/4.0/.

\section{ORCID iDs}

Joshua Jeong http://orcid.org/0000-0002-4130-468X

Helen 0 Pitchik http://orcid.org/0000-0002-5665-0884

Günther Fink http://orcid.org/0000-0001-7525-3668

\section{REFERENCES}

1 Lu C, Black MM, Richter LM. Risk of poor development in young children in low-income and middle-income countries: an estimation and analysis at the global, regional, and country level. Lancet Glob Health 2016;4:e916-22.

2 Bornstein $\mathrm{MH}$. Positive parenting and positive development in children. In: Lerner RM, Jacobs F, Wertlieb D, eds. Handbook of applied developmental science: promoting positive child, adolescent, and family development through research, policies, and programs. SAGE Publications, 2002: 187-209.

3 Britto PR, Lye SJ, Proulx K, et al. Nurturing care: promoting early childhood development. Lancet 2017;389:91-102.

4 Jeong J, Pitchik HO, Yousafzai AK. Stimulation interventions and parenting in low- and middle-income countries: a meta-analysis. Pediatrics 2018;141:e20173510.

5 Aboud FE, Yousafzai AK. Global health and development in early childhood. Annu Rev Psychol 2015;66:433-57.

6 Dowdall N, Melendez-Torres GJ, Murray L, et al. Shared Picture Book Reading Interventions for Child Language Development: A Systematic Review and Meta-Analysis. Child Dev 2020;91:e838-99.

7 Bakermans-Kranenburg MJ, van IJzendoorn MH, Juffer F. Less is more: meta-analyses of sensitivity and attachment interventions in early childhood. Psychol Bull 2003;129:195-215.

8 Jeong J, Franchett EE, Ramos C, et al. Parenting interventions to promote early child development in the first three years of life: a global systematic review and meta-analysis. revision 2021.

9 World Health Organization. Nurturing care for early childhood development: a framework for helping children survive and thrive to transform health and human potential. Geneva: World Health Organization, 2018.

10 Heckman JJ. Skill formation and the economics of investing in disadvantaged children. Science 2006;312:1900-2.

11 Nores M, Barnett WS. Benefits of early childhood interventions across the world: (under) investing in the very young. Econ Educ Rev 2010;29:271-82. 
12 Fergusson DM, Boden JM, Horwood LJ. Nine-year follow-up of a home-visitation program: a randomized trial. Pediatrics 2013;131:297-303.

13 Nordhov SM, Rønning JA, Dahl LB, et al. Early intervention improves cognitive outcomes for preterm infants: randomized controlled trial. Pediatrics 2010;126:e1088-94.

14 Eckenrode J, Campa M, Luckey DW, et al. Long-term effects of prenatal and infancy nurse home visitation on the life course of youths: 19-year follow-up of a randomized trial. Arch Pediatr Adolesc Med 2010;164:9-15.

15 McCormick MC, Brooks-Gunn J, Buka SL, et al. Early intervention in low birth weight premature infants: results at 18 years of age for the infant health and development program. Pediatrics 2006;117:771-80.

16 Campbell FA, Pungello EP, Burchinal M, et al. Adult outcomes as a function of an early childhood educational program: an Abecedarian project follow-up. Dev Psychol 2012;48:1033-43.

17 Belfield CR, Nores M, Barnett S. The high/scope Perry preschoo program cost-benefit analysis using data from the age-40 followup. Journal of Human Resources 2006;41:162-90.

18 van Aar J, Leijten P, Orobio de Castro B, et al. Sustained, fadeout or sleeper effects? A systematic review and meta-analysis of parenting interventions for disruptive child behavior. Clin Psychol Rev 2017;51:153-63.

19 Grantham-McGregor SM, Powell CA, Walker SP, et al. Nutritional supplementation, psychosocial stimulation, and mental development of stunted children: the Jamaican study. Lancet 1991;338:1-5.

20 Gertler P, Heckman J, Pinto R, et al. Labor market returns to an early childhood stimulation intervention in Jamaica. Science 2014;344:998-1001.

21 Walker SP, Chang SM, Powell CA, et al. Effects of early childhood psychosocial stimulation and nutritional supplementation on cognition and education in growth-stunted Jamaican children: prospective cohort study. Lancet 2005;366:1804-7.

22 Attanasio OP, Fernández C, Fitzsimons EOA, et al. Using the infrastructure of a conditional cash transfer program to deliver a scalable integrated early child development program in Colombia: cluster randomized controlled trial. BMJ 2014;349:g5785

23 Muhoozi GKM, Atukunda P, Diep LM, et al. Nutrition, hygiene, and stimulation education to improve growth, cognitive, language, and motor development among infants in Uganda: a cluster-randomized trial. Matern Child Nutr 2018;14:e12527.

24 Yousafzai AK, Rasheed MA, Rizvi A, et al. Effect of integrated responsive stimulation and nutrition interventions in the lady health worker programme in Pakistan on child development, growth, and health outcomes: a cluster-randomised factorial effectiveness trial. Lancet 2014;384:1282-93.

25 Chang SM, Grantham-McGregor SM, Powell CA, et al. Integrating a parenting intervention with routine primary health care: a cluster randomized trial. Pediatrics 2015;136:272-80.

26 Atukunda P, Muhoozi GKM, van den Broek TJ, et al. Child development, growth and microbiota: follow-up of a randomized education trial in Uganda J Glob Health 2019:9:010431.

27 Grantham-McGregor SM, Walker SP, Chang SM, et al. Effects of early childhood supplementation with and without stimulation on later development in stunted Jamaican children. Am J Clin Nutr 1997;66:247-53.

28 Cooper PJ, Tomlinson M, Swartz L, et al. Improving quality of mother-infant relationship and infant attachment in socioeconomically deprived community in South Africa: randomised controlled trial. BMJ 2009;338:b974.

29 Atukunda P, Muhoozi GKM, Westerberg AC, et al. Nutrition, hygiene and stimulation education for impoverished mothers in rural Uganda: effect on maternal depression symptoms and their associations to child development outcomes. Nutrients 2019;11:1561.

30 Yousafzai AK, Obradović J, Rasheed MA, et al. Effects of responsive stimulation and nutrition interventions on children's development and growth at age 4 years in a disadvantaged population in Pakistan: a longitudinal follow-up of a clusterrandomised factorial effectiveness trial. Lancet Glob Health 2016;4:e548-58.

31 Smith JA, Chang SM, Lopez Boo F, et al. Are benefits from a parenting intervention delivered through the health services sustainable? follow-up of a randomized evaluation in Jamaica. Acad
Pediatr 2021. doi:10.1016/j.acap.2021.01.003. [Epub ahead of print: 09 Jan 2021]

32 Walker SP, Chang SM, Younger N, et al. The effect of psychosocial stimulation on cognition and behaviour at 6 years in a cohort of term, low-birthweight Jamaican children. Dev Med Child Neuro 2010;52:e148-54.

33 Walker SP, Grantham-Mcgregor SM, Powell CA et al. Effects of growth restriction in early childhood on growth, $I Q$, and cognition at age 11 to 12 years and the benefits of nutritional supplementation and psychosocial stimulation. J Pediatr 2000;137:36-41.

34 Chang SM, Walker SP, Grantham-McGregor S, et al. Early childhood stunting and later behaviour and school achievement. J Child Psychol Psychiatry 2002;43:775-83.

35 Yousafzai AK, Rasheed MA, Rizvi A, et al. Parenting skills and emotional availability: an RCT. Pediatrics 2015;135:e1247-57.

36 Walker SP, Chang SM, Powell CA, et al. Psychosocial intervention improves the development of term low-birth-weight infants. J Nutr 2004;134:1417-23.

37 Murray L, Cooper P, Arteche A, et al. Randomized controlled trial of a home-visiting intervention on infant cognitive development in periurban South Africa. Dev Med Child Neurol 2016;58:270-6.

38 Barlow J, Bergman H, Kornør H, et al. Group-based parent training programmes for improving emotional and behavioural adjustment in young children. Cochrane Database Syst Rev 2016;8:CD003680.

39 Barnett WS. Effectiveness of early educational intervention. Science 2011;333:975-8.

40 Yousafzai AK, Aboud F. Review of implementation processes for integrated nutrition and psychosocial stimulation interventions. Ann N Y Acad Sci 2014;1308:33-45.

41 Walker SP, Wachs TD, Grantham-McGregor S, et al. Inequality in early childhood: risk and protective factors for early child development. Lancet 2011;378:1325-38.

42 Bailey D, Duncan GJ, Odgers CL, et al. Persistence and Fadeout in the impacts of child and adolescent interventions. J Res Educ Eff 2017;10:7-39.

43 Black MM, Walker SP, Fernald LCH, et al. Early childhood development coming of age: science through the life course. Lancet 2017;389:77-90

44 Obradović J, Yousafzai AK, Finch JE, et al. Maternal scaffolding and home stimulation: key mediators of early intervention effects on children's cognitive development. Dev Psychol 2016;52:1409-21.

45 Jeong J, Obradović J, Rasheed M, et al. Maternal and paternal stimulation: Mediators of parenting intervention effects on preschoolers' development. J Appl Dev Psychol 2019;60:105-18.

46 Smith JD, Cruden GH, Rojas LM, et al. Parenting interventions in pediatric primary care: a systematic review. Pediatrics 2020;146:e20193548.

47 Clarke A, Clarke AM. ?Sleeper effects? in development: Fact or artifact? Developmental Review 1981;1:344-60.

48 Schweinhart LJ, Montie J, Xiang Z. Lifetime effects: the High/Scope Perry preschool study through age 40. Ypsilanti, MI: High/Scope Press, 2005.

49 Rempel JK, Rempel LA, Hoa DTP, et al. Parenting teamwork: the impact of a Fathering intervention on mothers and infants in Vietnam. Child Dev 2020;91:e345-64.

50 Singla DR, Kumbakumba E, Aboud FE. Effects of a parenting intervention to address maternal psychological wellbeing and child development and growth in rural Uganda: a community-based, cluster randomised trial. Lancet Glob Health 2015;3:e458-69.

51 Walker SP, Chang SM, Powell CA, et al. Effects of psychosocial stimulation and dietary supplementation in early childhood on psychosocial functioning in late adolescence: follow-up of randomised controlled trial. BMJ 2006;333:472.

52 Walker SP, Chang SM, Vera-Hernández M, et al. Early childhood stimulation benefits adult competence and reduces violent behavior. Pediatrics 2011;127:849-57.

53 St Clair MC, Skeen S, Marlow M, et al. Relationships between concurrent language ability and mental health outcomes in a South African sample of 13-year-olds. PLoS One 2019;14:e0221242.

54 Andrew A, Attanasio O, Fitzsimons E, et al. Impacts 2 years after a scalable early childhood development intervention to increase psychosocial stimulation in the home: a follow-up of a cluster randomised controlled trial in Colombia. PLoS Med 2018;15:e1002556. 